\title{
Efficient sorting of Bessel beams
}

\author{
T. Mhlanga ${ }^{\mathrm{a}, \mathrm{b}}$, A. Dudley*a ${ }^{* \mathrm{a}}$ A. McDonald ${ }^{\mathrm{a}}$, F. S. Roux ${ }^{\mathrm{a}}$, M. Lavery $^{\mathrm{c}}$, M. Padgett ${ }^{\mathrm{c}}$ and A. Forbes ${ }^{\mathrm{a}, \mathrm{b}, \mathrm{d}}$ \\ ${ }^{\mathrm{a} C S I R}$ National Laser Centre, PO Box 395, Pretoria 0001, South Africa; ${ }^{\mathrm{b}}$ Stellenbosch University, \\ Private Bag X1, Matieland 7602, South Africa; ${ }^{\mathrm{c} D e p a r t m e n t ~ o f ~ P h y s i c s ~ a n d ~ A s t r o n o m y, ~ U n i v e r s i t y ~}$ \\ of Glasgow, Glasgow, UK; ' ${ }^{\mathrm{d}}$ School of Physics, University of KwaZulu-Natal, Private Bag X54001, \\ Durban 4000, South Africa
}

\begin{abstract}
A procedure to efficiently sort orbital angular momentum (OAM) states of light, by performing a Cartesian to log-polar coordinate transformation which translates helically phased beams into a transverse phase gradient, currently exists ${ }^{1}$. We implement this mode transformer, which comprises of two custom refractive optical elements ${ }^{2}$, to efficiently sort Bessel beams carrying OAM. Introducing two cylindrical lenses, allows the focusing of each of the input OAM Bessel states to a different lateral position in the Fourier plane and separates the radial wave-vectors in the image-plane. We demonstrate the concept by separating over forty OAM states and radial wave-vectors.
\end{abstract}

Keywords: Bessel beams, orbital angular momentum, sorting

\section{INTRODUCTION}

Numerous publications have been dedicated to optical fields which carry orbital angular momentum (OAM), ranging from Laguerre-Gaussian ${ }^{3}$, Bessel-Gauss ${ }^{4}$ and Airy beams ${ }^{5}$. Each of these fields has an azimuthal angular dependence of $\exp (i l \theta)^{3,6-8}$ where $l$ is the azimuthal index and $\theta$ is the azimuthal angle.

In this work we are interested in higher-order Bessel beams as an OAM-carrier as these fields offer other useful properties such as their ability to propagate diffraction-free over a finite distance ${ }^{9-13}$ and reconstruct after encountering an obstacle $^{14-16}$. The Bessel basis has been measured in entanglement and shown an increased spiral bandwidth ${ }^{17}$. Exploiting these properties of Bessel beams will make them very useful in the field of long-range, broad bandwidth communication systems. However, in order for these fields to be a success in the area of optical communication, efficient techniques for extracting the information they carry (i.e. their azimuthal mode indices) need to exist.

The 'fork' diffraction grating can be used to couple light of a particular OAM state into a single-mode fibre ${ }^{8}$, but this requires that one must test for all possible states simultaneously. Attempts to develop more complicated holograms which test multiple states have been made ${ }^{18,19}$, however their efficiency is inversely proportional to the number of states being sampled. An alternative setup that does not alter the OAM state during the measurement procedure is a Mach-Zehnder interferometer with two Dove prisms in each arm ${ }^{20,21}$, which sorts odd and even OAM states into two separate ports. Recently it has been demonstrated that two spatial light modulators (or refractive optical elements) in conjunction with a lens can be used to convert the OAM state of light to a specified lateral position ${ }^{1,2}$.

In this paper we make use of the above mentioned refractive optical elements to extract the information encoded in higher-order Bessel beams ${ }^{22}$. We first show that we can resolve forty-one OAM states and forty-one radial components of our higher-order Bessel beams separately. The channel capacity of the system to separate the OAM states of Bessel beams parallels that of the detection of the OAM states in Laguerre-Gaussian (LG) modes. By introducing cylindrical lenses into the optical setup, we illustrate that one is able to extract both the OAM states and radial components of the modes simultaneously.

*adudley@csir.co.za; phone +27 (0)12 8413741

Complex Light and Optical Forces VII, edited by Jesper Glückstad, David L. Andrews, Enrique J. Galvez, Proc. of SPIE Vol. 8637, 86371C - (c) 2013 SPIE · CCC code: 0277-786X/13/\$18 - doi: 10.1117/12.2004305 


\section{CONCEPT AND EXPERIMENTAL SETUP}

In this work we make use of Bessel beams as our OAM-carrying bases functions, which are characterized by an azimuthal mode index, $l$, and a radial component, $k_{r}$, as follows

$$
u(r, \theta, z=0)=J_{l}\left(k_{r} r\right) \exp (i l \theta) .
$$

$k_{r}$, is the transverse wave number and is defined as $k_{r}=k \sin \alpha$, where $k=2 \pi / \lambda$ and $\alpha$ is the opening angle of the cone on which the waves propagate.

The Fourier transform of the Bessel field, defined in Eq. (1) is described by an annular ring,

$$
\Im[u(r, \theta)]=\left\{\begin{array}{cc}
\exp (i l \theta) & R-\Delta \leq r \leq R+\Delta \\
0 & \text { elsewhere }
\end{array},\right.
$$

where $R$ is the radius of the annular ring and $\Delta$ is half its width. The radial component, $k_{r}$, is related to the radius of the annular ring, $R$, as $k_{r}=k \sin (R / f)$, where $f$ is the focal length of the Fourier transforming lens. It is these OAM-carrying fields, of differing radial components, whose OAM $(l)$ and radial information $(R)$ we will experimentally extract.

The technique that is employed for the measurement of the OAM spectrum and the radial components of the annular ring fields, consists of two refractive optical elements ${ }^{2}$ which map a position in an input plane $(x, y)$ to a position in the output plane $(v, u)$ by a conformal mapping ${ }^{2,23,24}$

$$
v=a \arctan \left(\frac{y}{x}\right)
$$

and

$$
u=-a \ln \left(\frac{R}{b}\right)
$$

Equation 4 illustrates that the vertical coordinate varies as a function of the radial component, $R$. The first refractive element which performs the log-polar mapping as given in Eqs (3) and (4), has a phase profile given by

$$
\phi_{1}(x, y)=\frac{a}{f(n-1)}[y \arctan \left(\frac{y}{x}\right)-x \ln \left(\frac{\sqrt{x^{2}+y^{2}}}{b}\right)+x-\frac{1}{a} \underbrace{\left(\frac{1}{2}\left(x^{2}+y^{2}\right)\right)}_{\text {lens term }}] .
$$

$f$ is the focal length of the lens integrated in the refractive optical element and $n$ its refractive index. The parameter $a$ controls the width of the azimuthal projection on the second refractive optical element, where $a=d / 2 \pi$ and $d$ is the length of the second refractive optical element along the $y$-axis; $b$ controls the scaling of the radial component and may be chosen independently of $a$.

The second refractive optical element is placed at a distance of $f$ behind the first and corrects any unwanted phase distortions associated with the first refractive optical element. It has a phase profile of

$$
\phi_{2}(x, y)=-\frac{a b}{f(n-1)}[\exp \left(-\frac{u}{a}\right) \cos \left(\frac{v}{a}\right)-\frac{1}{a b} \underbrace{\left(\frac{1}{2}\left(x^{2}+y^{2}\right)\right)}_{\text {lens term }}] \text {. }
$$

A schematic of the concept, where the above mentioned refractive optical elements are used to resolve both the azimuthal and radial components, is given in Fig. 1. 


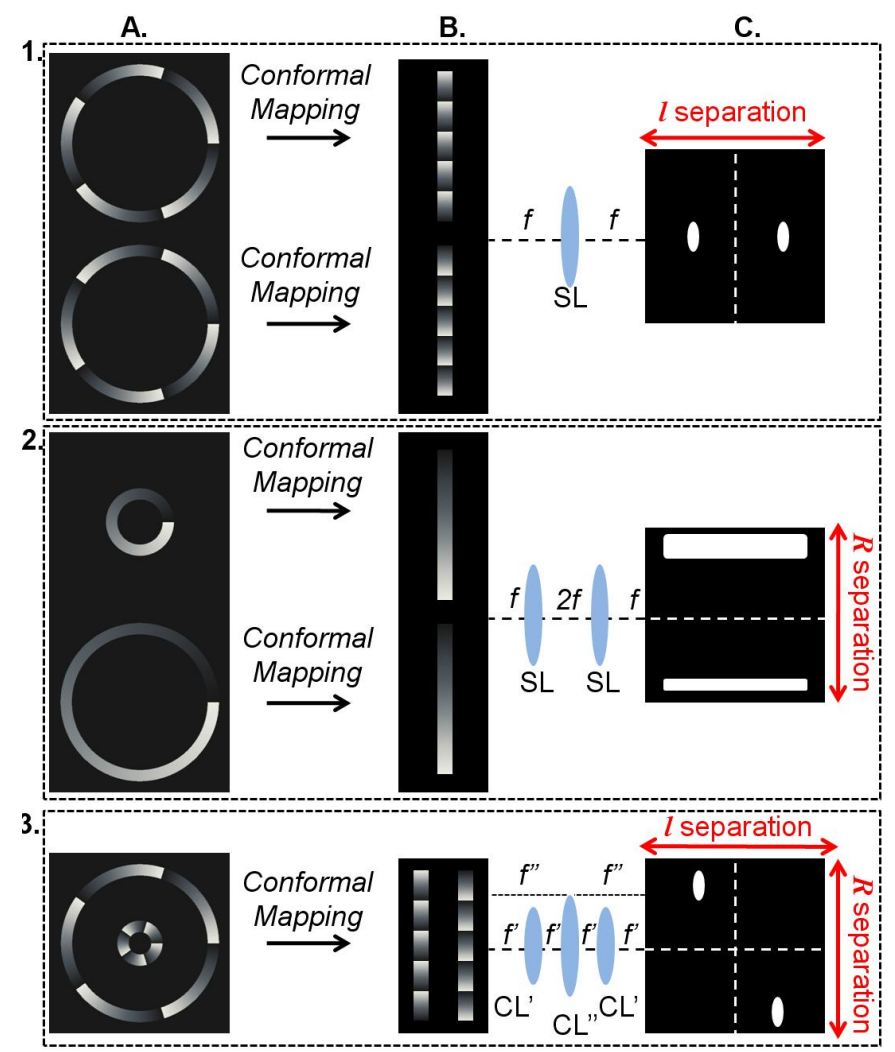

Fig. 1. Case 1, column A: Annular rings of the same radius but different azimuthal components $(l=-5$ and $l=+5)$ are mapped to transverse momentum modes represented by the linear phase variations in column B. A spherical lens (SL) arranged in a 2- $f$ configuration maps the transverse momentum modes to unique $x$-coordinates in column C. Case 2 , column A: Annular rings of the same azimuthal components $(l=+1)$ but different radii are mapped to transverse momentum modes represented by the linear phase variations in column B. Spherical lenses (SL) arranged in a 4- $f$ configuration image the transverse momentum modes to unique $y$-coordinates in column C. Case 3, column A: Superimposed annular rings of different radii and different azimuthal components $(l=-5$ and $l=+5)$ are mapped to transverse momentum modes represented by the linear phase variations in column B. Cylindrical lenses (CL) arranged in a 4- $f$ configuration map the transverse momentum modes to unique $x$ - and $y$-coordinates in column $\mathrm{C}$, respectively.

The conformal mapping that is described by Eqs (3) and (4) and achieved by the phase profile given in Eq. (5) is depicted to occur between columns A and B in Fig. 1. The annular ring structure possessing a particular azimuthal phase variation of a particular radial component is mapped to a linear phase variation. Each of the cases represented in Fig. 1 resembles the concept for separating azimuthal components (case 1), radial components (case 2) and both azimuthal and radial components simultaneously (case 3). In case 1, two annular rings possessing the same radial components, but different azimuthal orders $(l=-5$ and $l=+5)$, given in column A, are transformed to a linear phase variation as represented in column B. A spherical lens is placed after the second refractive optical element to focus the transformed beam to a specified spot in the focal plane, represented in column $\mathrm{C}$. The position of the spot varies along the $x$-direction as a function of the incident azimuthal order and is defined by

$$
\Delta_{x}=\frac{\lambda f}{d} l .
$$

Case 2 represents the concept for resolving the radial component of the incident beam. Two annular rings possessing the same azimuthal component $(l=+1)$, but different radial components as given in column A, are transformed to a linear phase variation represented in column B. By arranging two spherical lenses in a 4- $f$ configuration, the unravelled linear phase variation is imaged to the plane represented in column $\mathrm{C}$ and varies along the $y$-direction as a function of the incident radial component as defined by Eq. (3). 


$$
\Delta_{y}=a \ln \left(\frac{R}{b}\right)
$$

To combine both of the techniques represented in cases 1 and 2 , to simultaneously resolve the azimuthal and radial components to $x$ - and $y$-coordinates respectively, cylindrical lenses are arranged in a 4-f configuration, as depicted in case 3. The $x$-axis at the plane contained in column B is Fourier transformed to a specified $x$-position at column $\mathrm{C}$ (achieved by cylindrical lens CL"), while cylindrical lenses CL' image the $y$-axis at column B's plane to that in column C. This results in the transverse momentum modes at column $\mathrm{B}$ being transformed to a specified position on the $x$-axis at column $\mathrm{C}$, while simultaneously imaging the unravelled annular ring to a specified position on the $y$-axis. The example given in case 3 consists of a superposition of two annular rings that are resolved to two different spots, whose $x$ - and $y$ coordinates are related to their azimuthal and radial components respectively.

The experimental setup is presented in Fig. 2 (a). A HeNe laser was expanded through a telescope (lenses L1 and L2) to illuminate the liquid crystal display of a spatial light modulator (SLM). The SLM was programmed to produce the Fourier transform of Bessel beams of various azimuthal and radial orders which were imaged onto the entrance of the mode sorter, located at the first refractive optical element (R1). To produce the Fourier transform of a Bessel beam, an annular ring containing a particular azimuthal order was encoded onto the SLM; such an example is given in Fig. 2 (b). Alternating sets of pixels surrounding the annular ring were assigned phase values of 0 and $\pi$, producing an amplitude transmission of 0 on our phase-only SLM $^{25,26}$. The Fourier transform of these annular rings produce non-diffracting petal-like modes that have been studied in detail elsewhere ${ }^{27}$. 28 . The resulting annular rings were propagated through the mode sorter (denoted by refractive optical elements, R1 and R2), where R1 performed a log-polar mapping thus transforming the azimuthal modes to transverse momentum modes, while consequently mapping the radial component to a unique latitude. The second refractive optical element (R2) corrected the unwanted phase associated with R1. The phase profiles of the refractive optical elements R1 and R2, obtained from Eqs (5) and (6), are presented in Figs 2 (c) and 2 (d) respectively.

(a)

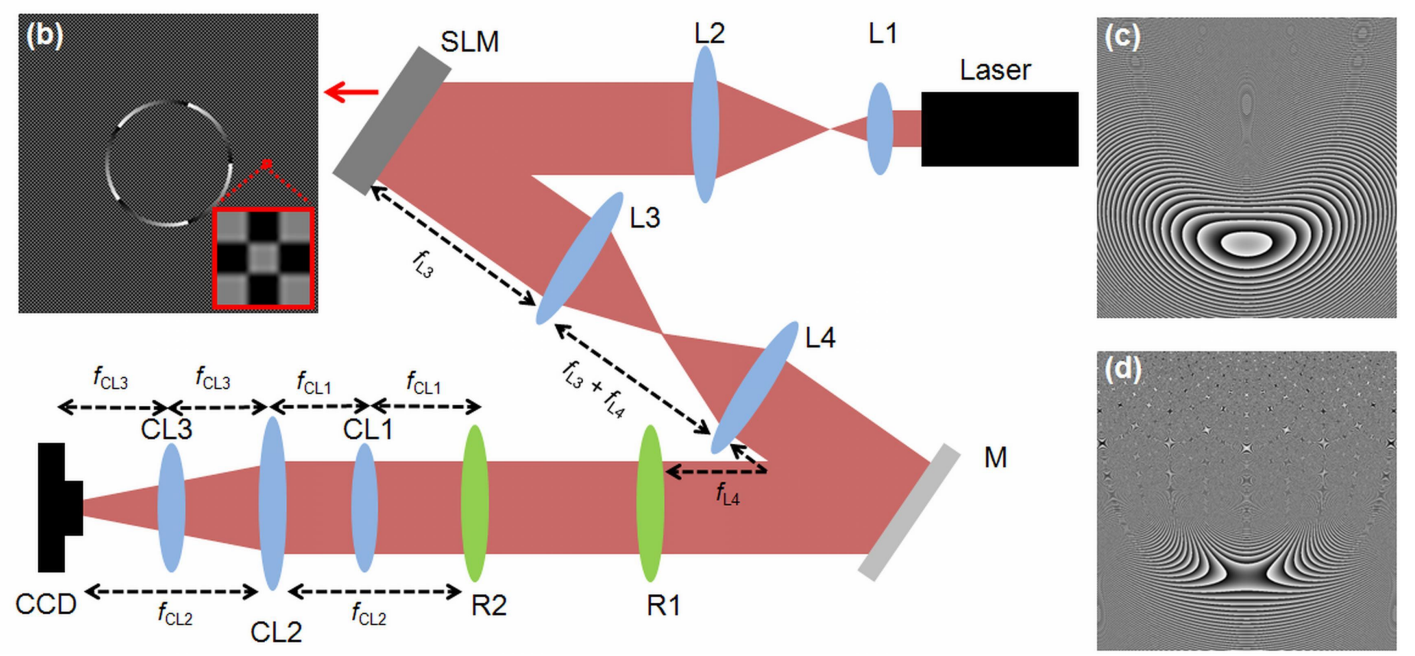

Fig. 2. (a) Schematic of the experimental setup for efficiently sorting the azimuthal and radial components of Bessel beams. $\mathrm{L}$, lens $\left(f_{\mathrm{L} 1}=15 \mathrm{~mm}, f_{\mathrm{L} 2}=125 \mathrm{~mm}, f_{\mathrm{L} 3}=500 \mathrm{~mm}, f_{\mathrm{L} 4}=500 \mathrm{~mm}\right)$; SLM, spatial light modulator; M, mirror; R, refractive optical element; CL, cylindrical lens ( $\mathrm{f}_{\mathrm{CL} 1}=50 \mathrm{~mm}$ (orientated to focus the $y$-axis), $\mathrm{f}_{\mathrm{CL} 2}=100 \mathrm{~mm}$ (orientated to focus the $x$ axis), $\mathrm{f}_{\mathrm{CL} 3}=50 \mathrm{~mm}$ (orientated to focus the $y$-axis)); CCD, CCD camera. (b) The phase profile encoded on the SLM and a zoomed-in insert of the alternating phase values surrounding the ring-slit. (c) and (d) The phase profiles of the refractive optical elements R1 and R2, respectively.

\section{EXPERIMENTAL RESULTS AND DISCUSSION}

Annular rings of radius, $R=1320 \mu \mathrm{m}$, having azimuthal orders ranging from $l=-20$ to +20 were generated and directed through the mode sorter. An example of a hologram, for an azimuthal order of $l=-5$, encoded on the SLM and the annular ring produced at the plane of R1 together with its Fourier transform are given in the first row of Fig. 3 ((a) - (c), respectively). The unravelled transverse momentum mode at the plane of $\mathrm{R} 2$ was focused by a cylindrical lens having a 
focal length of $f=100 \mathrm{~mm}$, producing an elongated lateral spot shown in Fig. 3 (d), with its theoretical prediction given alongside in Fig. 3 (e). Due to the alignment sensitivity of the cylindrical lenses the agreement between the experimentally recorded lateral spot (Fig. 3 (d)) and its theoretical prediction (Fig. 3 (e)) is not very good. We illustrate that the result can be improved by replacing the three cylindrical lenses with a single spherical lens $(f=250 \mathrm{~mm})$, as in case 1 of Fig. 1, producing the experimentally recorded lateral spot in Fig. 3 (f) which is in very good agreement with its theoretical prediction in Fig. 3 (g). The white dotted line represents the position for the lateral spot for an azimuthal mode of $l=0$.

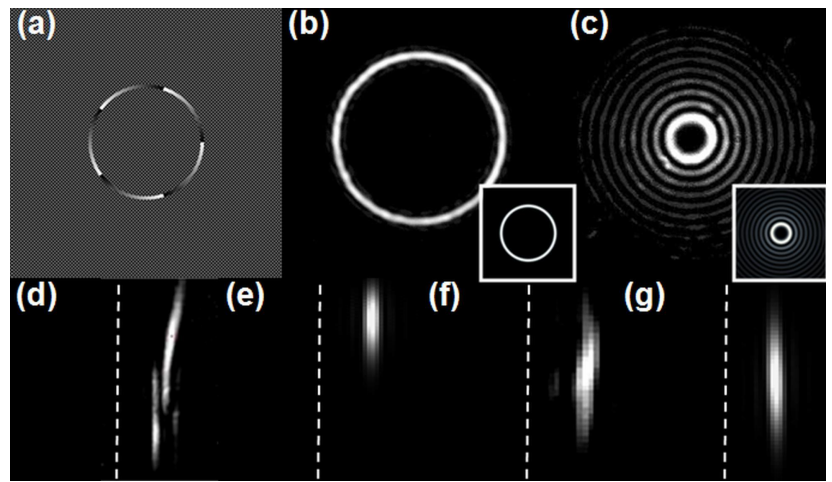

Fig. 3. (a) The hologram, (b) annular ring, and (c) Bessel beam for a radial component of $R=1320 \mu \mathrm{m}$ and an azimuthal component of $l=-5$. The theoretical results are given as inserts. (d) The experimentally recorded and (e) theoretically calculated lateral spot when cylindrical lenses are used (as in case 3 of Fig. 1). (f) The experimentally recorded and (g) theoretically calculated lateral spot when a spherical lens $(f=250 \mathrm{~mm})$ is used (as in case 1 of Fig. 1).

The position of the spots produced by incoming azimuthal modes ranging for $l=-20$ to +20 were measured and are given in Fig. 4 (a). There is very good agreement between the experimentally measured positions (red circles) and the theoretical expected positions (black curve), calculated with the use of Eq. (7). Apertures in the detector plane were centred on the expected spot positions and the intensity in each of the apertures was measured for each input mode. The relative fraction of the OAM state was determined and is presented in Fig. 4 (b).
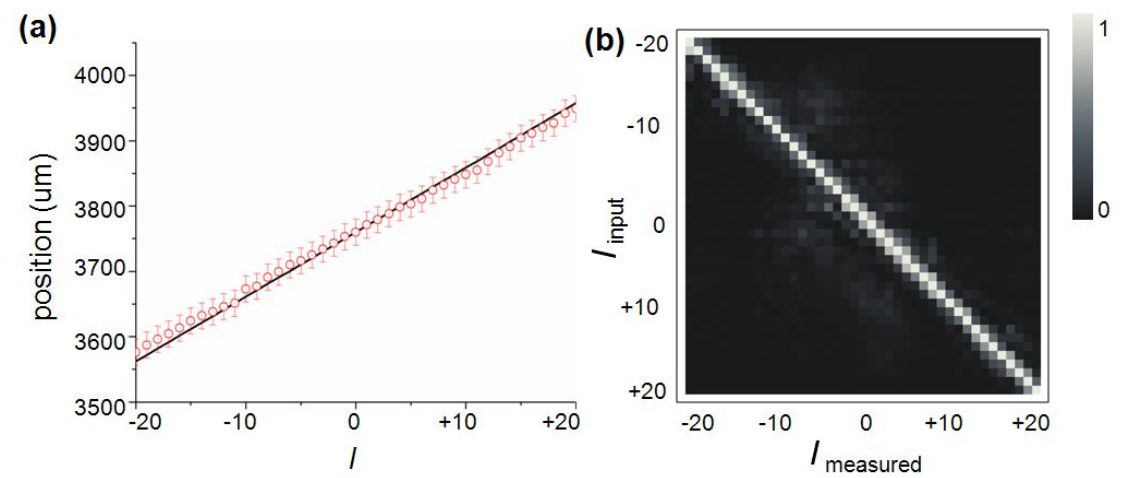

Fig. 4. (a) The position of the spot produced at the plane of the CCD as a function of the incoming azimuthal order, $l$. (b) Relative fractions of the intensity at each detector position for incoming OAM states ranging from $l=-20$ to +20 (for $R=$ $1320 \mu \mathrm{m})$.

The radial component of the annular ring entering the mode sorter was varied from values of $R=120$ to 3240 $\mu \mathrm{m}$. An example of a hologram and its corresponding annular ring and Fourier transform is given in the first row of Fig. 5 ((a) - (c)). The transformed unravelled annular rings, at the plane of R2, were imaged onto the CCD, with the use of cylindrical lenses CL1 and CL3 and an example is depicted in Fig. 5 (d), together with its theoretical prediction in Fig. 5 (e). Due to the alignment sensitivity of the cylindrical lenses the agreement between the experimentally recorded lateral spot (Fig. 5 (d)) and its theoretical prediction (Fig. 5 (e)) is not very good. We illustrate that the result can be improved by replacing the three cylindrical lenses with two spherical lenses $(f=250 \mathrm{~mm})$, as in case 2 of Fig. 1, imaging the 
unravelled transverse momentum mode to the CCD as in Fig. 5 (f) which is in very good agreement with its theoretical prediction in Fig. 5 (g). The white dotted line marks a common reference on the CCD image.

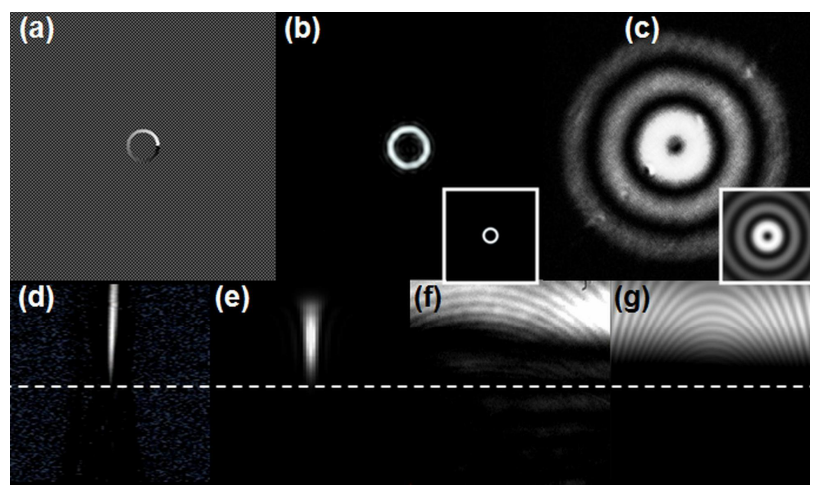

Fig. 5. (a) The hologram, (b) annular ring, and (c) Bessel beam for a radial component of $R=360 \mu \mathrm{m}$ and an azimuthal component of $l=+1$. The theoretical results are given as inserts. (d) The experimentally recorded and (e) theoretically calculated lateral spot when cylindrical lenses are used (as in case 3 of Fig. 1). (f) The experimentally recorded and (g) theoretically calculated unravelled transverse momentum mode when two spherical lenses $(f=250 \mathrm{~mm})$ are used (as in case 2 of Fig. 1).

The position of the spot in the detector plane was plotted as a function of the radius of the annular ring and is given in Fig. 6 (a). There is fairly ood agreement between the experimentally measured positions (red circles) and the theoretical expected positions (black curve), calculated with the use of Eq. (8). Similarly in detecting the radial components, apertures in the detector plane are centred on the expected line positions and relative fractions of the radial spectrum for various input modes are determined and presented in Fig. 6 (b). There is a slight overlap of the unravelled lines between neighbouring radial coordinates, illustrated by the off-diagonal elements in Fig. 6 (b). The spacing between neighbouring radial components is $80 \mu \mathrm{m}$ and it is evident from Fig. 6 (b) that if this spacing is doubled, the cross-talk between neighbouring radial modes will be drastically diminished.
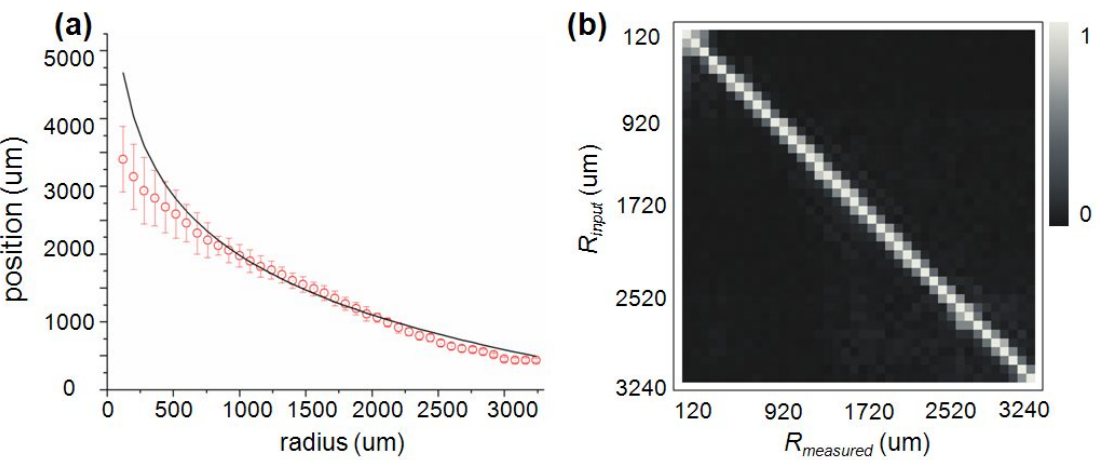

Fig. 6. (a) The position of the spot produced at the plane of the CCD as a function of the incoming radial component, $R$. (b) Relative fractions of the intensity at each detector position for incoming radial components ranging from $R=120$ to 3220 $\mu \mathrm{m}($ for $l=+1)$.

Multiple annular rings were also directed through the mode sorter and an example is presented in Fig 7. An example of a hologram containing two annular rings of differing radii and azimuthal modes is given in Fig. 7 (a) together with its corresponding annular ring field and Fourier transform (superimposed Bessel beams) in Figs 7 (b) and (c), respectively. The separation of the two azimuthal and two radial components, when using cylindrical lenses CL1, CL2 and CL3, is depicted in Fig. 7 (d) accompanied with the theoretical prediction in Fig. 7 (e), illustrating that the mode sorter is capable of distinguishing superimposed azimuthal modes. There is fairly good agreement in terms of the locations of the experimentally recorded lateral spots (Fig. 7 (d)) and their theoretical predictions (Fig. 7 (e)). However, the shape of the experimentally recorded lateral spots does not agree very well with their theoretical predictions due to the alignment sensitivity of the cylindrical lenses. 


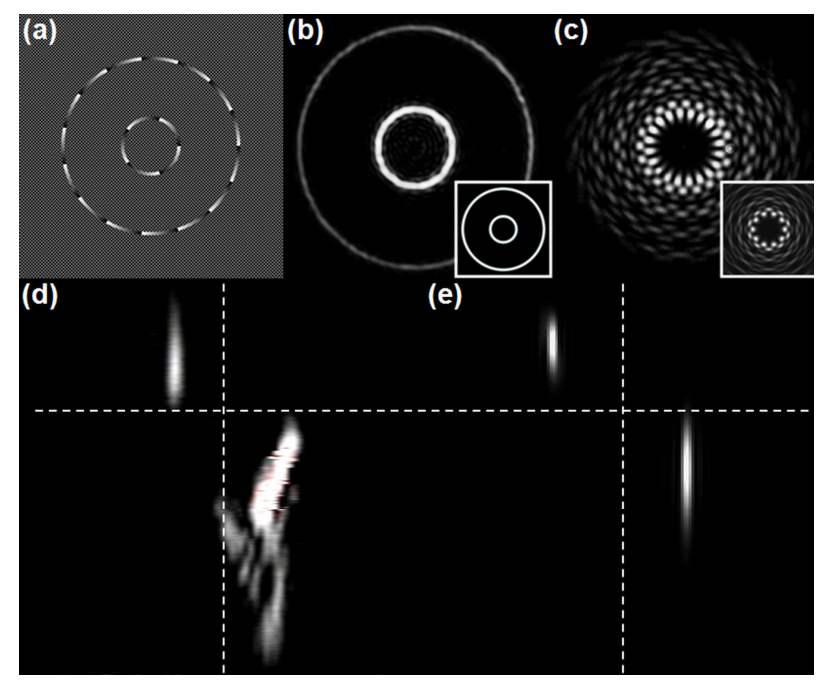

Fig. 7. (a) Digital hologram of multiple azimuthal and radial orders $R_{1}=680 \mu \mathrm{m}, R_{2}=2120 \mu \mathrm{m}, l_{1}=-5, l_{2}=+15$, and (b) the corresponding annular ring field and (c) Fourier transform (superimposed Bessel beams). The theoretical results are given as inserts. (d) The corresponding experimental and (e) theoretical spots produced at the plane of the CCD.

We also investigated the effect of misaligning the input mode $(l=+1$ and $R=1320 \mu \mathrm{m})$ by changing the angle of incidence by 0.002 radians with respect to the $x$ - and $y$-axes of the first refractive optical element, $\mathrm{R} 1$, and the result is presented in Fig. 8. Figure 8 (a) and (d) denote the lateral spot produced in the detector plane for an aligned annular ring $(l=+1$ and $R=1320 \mu \mathrm{m})$. Figures $8(\mathrm{~b})((\mathrm{e}))$ and (c) ((f)) contain the lateral spot when the angle of incidence of the input mode is adjusted with respects to the $x$-axis ( $y$-axis) by +0.002 radians and -0.002 radians, respectively, illustrating that a slight misalignment results in states 'leaking' into neighbouring states. This illustrates that although this device is ideal at measuring a large set of azimuthal and radial components simultaneously, it is very sensitive to misalignment of the incoming mode.

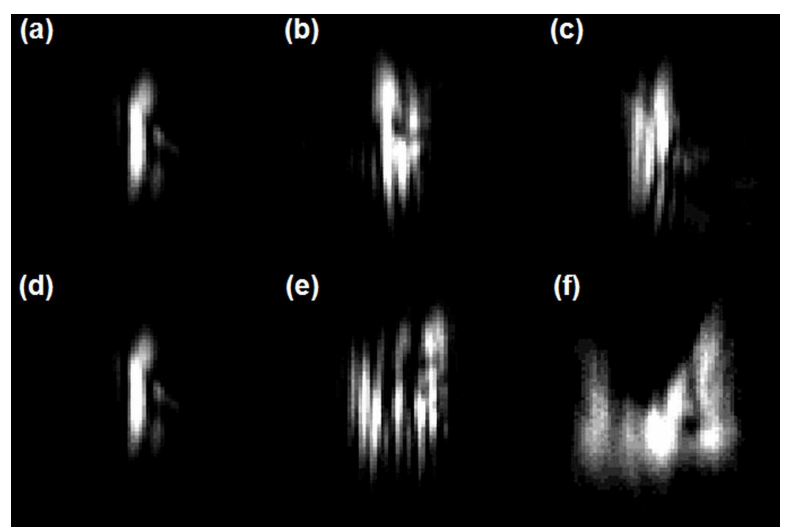

Fig. 8. The resulting lateral spot in the detector plane when the input mode is aligned (a) and (d); its angle of incidence is adjusted by +0.002 radians (b) and -0.002 radians (c) with respect to the $x$-axis; and adjusted by +0.002 radians (e) and 0.002 radians (f) with respect to the $y$-axis.

\section{CONCLUSION}

We have illustrated the separation of higher-order Bessel beams in both their azimuthal and radial bases. The ability to extract encoded information across two higher-dimensional state spaces will prove very useful in quantum communication and information systems. Although there is a slight overlap between neighbouring modes in separating both the OAM and radial modes, we suggest first separating the OAM modes into odd and even ports ${ }^{20,21}$ to decrease any cross-talk. 


\section{REFERENCES}

[1] Berkhout, G. C. G., Lavery, M. P. J., Courtial, J., Beijersbergen, M. W. and Padgett, M. J., "Efficient sorting of orbital angular momentum states of light,” Phys. Rev. Lett. 105(15), 153601 (2010).

[2] Lavery, M. P. J., Robertson, D. J., Berkhout, G. C. G., Love, G. D., Padgett, M. J. and Courtial, J., "Refractive elements for the measurement of the orbital angular momentum of a single photon," Opt. Express 20(3), 21102115 (2012).

[3] Beijersbergen, M. W., Allen, L., Van der Veen, H. E. L. O. and Woerdman, J. P., "Astigmatic laser mode converters and the transferof orbital angular momentum," Opt. Commun. 96, 123-132 (1993).

[4] Arlt, J. and Dholakia, K., "Generation of high-order Bessel beams by use of an axicon," Opt. Commun. 177, 297-301 (2000).

[5] Sztul, H. I. and Alfano R. R., "The Poynting vector and angular momentum of Airy beams," Opt. Express 16, 9411-9416 (2008).

[6] Allen, L., Beijersbergen, M. W., Spreeuw, R. J. C. and Woerdman, J. P., "Orbital angular momentum of light and the transformation of Laguerre-Gaussian laser modes," Phys. Rev. A 45, 8185-8189 (1992).

[7] He, H., Friese, M. E. J., Heckenberg, N. R. and Rubinsztein-Dunlop, H., "Direct observation of transfer of angular momentum to absorptive particles from a laser beam with a phase singularity,"Phys. Rev. Lett. 75, 826829 (1995).

[8] Mair, A., Vaziri, A., Weihs, G. and Zeilinger, A., "Entanglement of the orbital angular momentum states of photons," Nature 412, 313-316 (2001).

[9] Durnin J., Miceli, J. J. and Eberly, J. H., "Diffraction-free beams," Phys. Rev. Lett. 58(15), 1499-1501 (1987).

[10] Herman, R. M. and Wiggins, T. A., "Production and uses of diffractionless beams," J. Opt. Soc. Am. A 8(6), 932-942 (1991).

[11] Arlt, J. and Dholakia, K., "Generation of high-order Bessel beams by use of an axicon," Opt. Commun. 177(16), 297-301 (2000).

[12] Turunen, J., Vasara, A. and Friberg, A. T., "Holographic generation of diffraction-free beams," Appl. Opt. 27(19), 3959-3962 (1988).

[13] Vasara, A., Turunen, J. and Friberg, A. T., "Realization of general nondiffracting beams with computergenerated holograms," J. Opt. Soc. Am. A 6(11), 1748-1754 (1989).

[14] MacDonald, J. A., Boothroyd, S. A., Okamoto, T., Chrostowski, J. and Syrett, B. A., "Interboard optical data distribution by Bessel beam shadowing," Opt. Commun. 122, 169-177 (1996).

[15] Bouchal, Z., Wagner, J. and Chlup, M., "Self-reconstruction of a distorted nondiffracting beam," Opt. Commun. 151, $207-211$ (1998).

[16]Litvin, I., McLaren, M. and Forbes, A., "A conical wave approach to calculating Bessel-Gauss beam reconstruction after complex obstacles," Opt. Commun. 282(6), 1078-1082 (2009).

[17] McLaren, M., Agnew, M., Leach, J., Roux, F. S., Padgett, M. J., Boyd, R. W. and Forbes, A., "Entangled Bessel-Gaussian beams," Opt. Express 20(21), 23589-23597 (2012).

[18] Gibson, G., Courtial, J., Padgett, M., Vasnetsov, M., Pas'ko, V., Barnett, S. and Franke-Arnold, S., "Free-space information transfer using light beams carrying orbital angular momentum," Opt. Express 12, 5448-5456 (2004).

[19] Khonina, S. N., Kotlyar, V. V., Skidanov, R. V., Soifer, V. A., Laakkonen, P. and Turunen, J., "Gauss-Laguerre modes with different indices in prescribed diffraction orders of a diffractive phase element," Opt. Commun. 175, 301 (2000).

[20]Leach, J., Padgett, M. J., Barnett, S. M., Franke-Arnold, S. and Courtial, J., "Measuring the orbital angular momentum of a single photon," Phys. Rev. Lett. 88(25), 257901 (2002).

[21] Lavery, M., Dudley, A., Forbes, A., Courtial, J. and Padgett, M., "Robust interferometer for the routing of light beams carrying orbital angular momentum," New J. of Phys. 13(9), 093014 (2011).

[22] Dudley, A., Mhlanga, T., Lavery, M., McDonald, A., Roux, F. S., Padgett, M. and Forbes, A., "Efficient sorting of Bessel beams," Opt. Express 21(1), 165-171 (2013).

[23] Lavery, M. J. P., Berkhout, G. C. G., Courtial, J. and Padgett, M. J., "Measurement of the light orbital angular momentum spectrum using an optical geometric transformation," J. Opt. 13, 064006 (2011).

[24] Berkhout, G. C. G., Lavery, M. P. J., Beijersbergen, M. W. and Padgett, M. J., "Measuring orbital angular momentum superpositions of light by mode transformation," Opt. Lett. 36, 1863-1865 (2011). 
[25] Rop, R., Dudley, A., Lopez-Mariscal, C. and Forbes, A., "Measuring the rotation rates of superpositions of higher-order Bessel beams," J. Mod. Opt. 59(3), 259-267 (2012).

[26] López-Mariscal, C. and Helmerson, K., "Shaped nondiffracting beams," Opt. Lett. 35(8), 1215-1217 (2010).

[27] Vasilyeu, R., Dudley, A., Khilo, N. and Forbes, A., "Generating superpositions of higher-order Bessel beams," Opt. Express 17(26), 23389-23395 (2009).

[28] Rop, R., Litvin, I. A. and Forbes, A., "Generation and propagation dynamics of obstructed and unobstructed rotating orbital angular momentum-carrying Helicon beams," J. Opt. 14(3), 035702 (2012). 